\title{
Giant Fungated Locally Advanced Breast Carcinoma Responded to Hypofractionated Radiotherapy Combined with Apatinib: A Case Report and Literature Review
}

This article was published in the following Dove Press journal:

Cancer Management and Research

\author{
Hui Liu' ${ }^{1} *$ \\ Bailong Liu',* \\ Yunfei Ma' \\ Liang Guo ${ }^{2}$ \\ Di $\mathrm{Wu}^{3}$ \\ Aiping Shi $\mathbb{1 D}^{3}$ \\ Min Liu' \\ 'Department of Radiation Oncology, The \\ First Hospital of Jilin University, \\ Changchun I3002I, People's Republic of \\ China; ${ }^{2}$ Department of Pathology, The \\ First Hospital of Jilin University, \\ Changchun I3002I, People's Republic of \\ China; ${ }^{3}$ Department of Breast Surgery, \\ The First Hospital of Jilin University, \\ Changchun I3002I, People's Republic of \\ China
}

*These authors contributed equally to this work

Correspondence: Aiping Shi

Department of Breast Surgery, The First Hospital of Jilin University, I Xinmin

Street, Changchun I3002I, People's

Republic of China

Tel +86 I33-6430-8696

Email sap@jlu.edu.cn

Min Liu

Department of Radiation Oncology, The

First Hospital of Jilin University, I Xinmin

Street, Changchun I3002I, People's

Republic of China

Tel + 86 I58-0430-275 I

Email Imin99@jlu.edu.cn

\begin{abstract}
Locally advanced breast cancer (LABC) is frequently encountered in clinical practice. Primary systemic therapy is regarded as the cornerstone of LABC management to downstage the disease and enable surgery. However, multiple lines of systemic agents may fail to control tumor growth in a considerable number of patients, and few options remain available for such patients. Here, we present a case of triple-negative, right breast cancer that progressed aggressively despite 3 lines of standard chemotherapy. The patient suffered from severe skin ulceration, bleeding, pain, infection, and fungation. The small-molecular tyrosine kinase inhibitor (TKI) apatinib was initiated, which targets vascular endothelial growth factor receptor 2 (VEGFR2). The patient then underwent hypofractionated irradiation applied to the whole right breast at $40 \mathrm{~Gy} / 8 \mathrm{f}$. The tumor responded dramatically to this combination, and a near-complete remission (CR) response was achieved 2 months after irradiation. Our case is novel and instructional and demonstrated the efficacy and safety of hypofractionated irradiation combined with antiangiogenesis for the treatment of intractable LABC, shedding light on this difficult situation. In the near future, large-scale clinical trials will be initiated to further explore this issue.
\end{abstract}

Keywords: intractable locally advanced breast cancer, triple negative breast cancer, hypofraction, irradiation, antiangiogenesis

\section{Introduction}

Triple negative breast cancer (TNBC) accounts for $15 \%-20 \%$ of all invasive breast cancer (BC). ${ }^{1}$ Due to the lack of hormone receptor expression, including estrogen receptor (ER), progesterone receptor (PR), and human epidermal growth factor receptor 2 (HER-2) expression, chemotherapy is the mainstay of TNBC treatment. ${ }^{2}$ However, TNBC often progresses even after several lines of standard chemotherapy regimens. Currently, the management of TNBC can be complicated, and no consensus regarding the treatment of TNBC refractory to standard chemotherapy exists. Here, we describe a locally advanced TNBC that responded positively to hypofractionated radiotherapy plus apatinib, a vascular endothelial growth factor receptor 2 (VEGFR2) inhibitor following the failure to respond to multiple lines of intensive chemotherapy. Our case treatment strategy provided an option for achieving good short-term local control, which greatly alleviated patient suffering. Antiangiogenesis overcame radiation resistance, to a certain extent. Novel therapeutic avenues are 
urgently necessary to maintain TNBC patients free of distant metastases and to achieve durable local remission because TNBC has the high propensity to metastasize preferentially to the viscera and relapse. ${ }^{1}$

\section{Case Presentation}

A 67-year-old, Chinese woman was referred to a tertiary hospital in 2018 with a 2-year history of a mass in the right breast. The subsequent pathological analysis of biopsy tissue revealed invasive ductal breast carcinoma (IDBC) with immunohistochemical results of $\mathrm{ER}(-), \operatorname{PR}(-)$, androgen receptor $(\mathrm{AR})(-)$, HER2(3+), and $\mathrm{Ki} 67(20 \%+)$. Five cycles of primary systemic therapy with TA (Docetaxel, $120 \mathrm{mg}$, and epirubicin, $130 \mathrm{mg}$, for $21 \mathrm{~d}$ in each cycle) were performed. However, the tumor grew fiercely, and skin ulceration occurred. In December 2018, the patient presented to our hospital. Breast ultrasound showed a huge hypoechoic mass, measuring approximately $117 \mathrm{~mm} \times$ $45.6 \mathrm{~mm}$, located in the right breast. The pathological results of a second biopsy from the right breast lump further confirmed IDBC, but the immunohistochemical results demonstrated triple negativity, with $\mathrm{CK} 5 / 6(+)$ and $\mathrm{Ki67}(50 \%+)$ (Figure 1). The evaluation of bilateral axillary lymph nodes, lung, liver, and skeleton revealed no involvement of these tissues. Because the HER-2 immunohistochemistry result was once $3+$, and fluorescence in situ hybridization (FISH) results had not yet been reported, one cycle of TCH (Docetaxel, $110 \mathrm{mg}$, d2; Carboplatin, $550 \mathrm{mg}$, d2; and

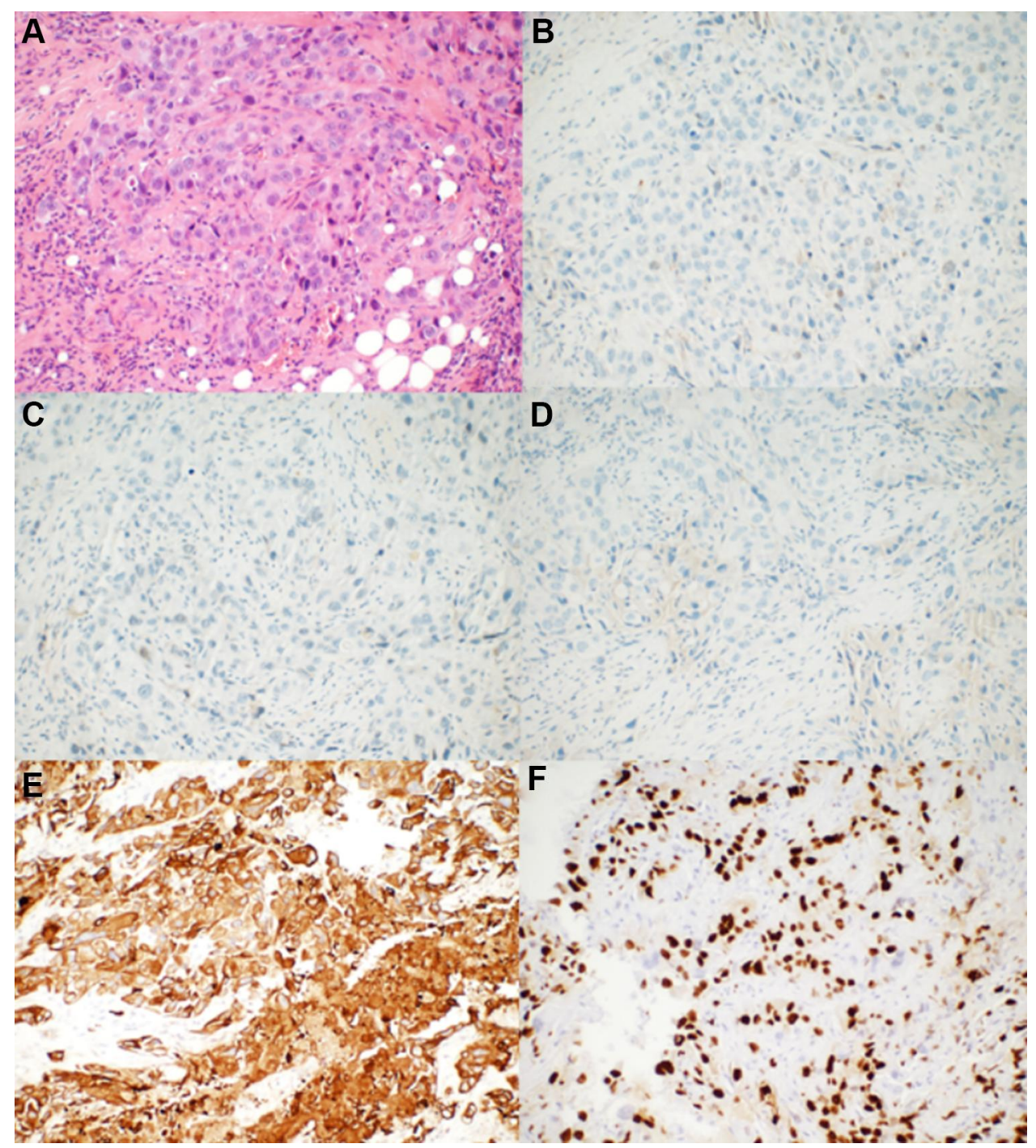

Figure I The pathological results of the rebiopsy pathology of the right breast tumor performed in our hospital. (A) Hematoxylin-eosin-stained sections revealed that the tumor cells grew in a solid and patchy infiltrating manner (original magnification: 200x). (B-D) ER, PR, and HER-2 were negative for neoplastic cells by immunohistochemical analysis (original magnification: 200x). (E) CK5/6 was strongly expressed by tumor cells (original magnification: 200x). (F) Ki-67 was expressed in the nuclei of approximately $50 \%$ of tumor cells (original magnification: $200 \times$ ). 
Trastuzumab, $400 \mathrm{mg}$, d1) was performed, but the response to treatment remained progressive disease (PD). The reevaluation of pathology at the tertiary hospital and further FISH analysis confirmed no amplification of the HER2 gene. Trastuzumab was suspended, and the chemotherapy regimen was switched to NP (Vinorelbine, $36 \mathrm{mg}, \mathrm{d} 1$, 8; Carboplatin, $500 \mathrm{mg}$, d1), which continued to have no effect. The ulcer spread rapidly throughout the entire right breast. Apatinib, at a dose of $250 \mathrm{mg}$ daily, was administered starting January 29, 2019, to March 3, 2019, resulting in a stable disease (SD) response.

After a multidisciplinary consultation, radical radiotherapy (RT) was recommended. At this time, the entire right breast had disappeared, replaced by a huge, fungated, cauliflower-like mass, measuring approximately $110 \mathrm{~mm} \times$ $95 \mathrm{~mm}$ with a foul smell (Figure 2A). A hypofractionated irradiation of $40 \mathrm{~Gy} / 8$ fractions was delivered to the tumor, using a biological effective dose (BED) of 60 Gy (Varian 21EX, Varian Corporation, USA). The detailed RT technique was as follows: three-dimensional conformal radiotherapy (3D-CRT), 6 MV (Eclipse 13.6 planning system, Varian Corporation, USA); the V20 and mean dose of the right lung were $7.03 \%$ and 392.4 cGy respectively. The patient's quality of life was improved dramatically, including reduced bleeding and pain and the cessation of the foul odor. After RT, she continued apatinib for 28 days, from March 16 to April 12, at a dose of $250 \mathrm{mg}$ daily. Then, apatinib was suspended because the patient could not afford the expense. Surprisingly, the huge tumor demonstrated a near-complete remission (CR) response at the 2-month follow-up after RT (Figure 2B). No hematologic toxicities, hypertension, proteinuria, or hand-foot syndrome occurred. The patient only complained of mild fatigue (grade 1) according to the Common Terminology Criteria for Adverse Events (CTCAE), V4.03, criteria. However, this patient passed away in August 2019 due to brain metastasis. At that time, recurrence in the chest wall was detected.

\section{Discussion}

The treatment for TBNC is challenging due to the lack of identified molecular targets, ${ }^{3}$ and TNBC tends to be characterized with as highly proliferative ${ }^{4}$ with rapid progression. ${ }^{5}$ Recently, few breakthroughs have been detailed in this field, which remains restricted to the application of conventional, cytotoxic chemotherapies. However, in our patient, the tumor progressed fiercely, despite the use of multiple lines of standard chemotherapy agents, such as anthracycline, taxanes, and platinum. Under such circumstances, disease management becomes rather difficult, with limited options.

$\mathrm{RT}$ plays an irreplaceable role in the treatment of $\mathrm{BC}$; however, the role of RT in LABC requires further exploration. Yee et al retrospectively reviewed 43 inoperable LABC cases, although TNBC was excluded from this analysis. RT delivered to the breast, chest wall, or related regional lymph nodes contributed to a median local progression-free survival (PFS) of 12 months. Eighty-four percent of the cohort demonstrated a good response to RT, with tumor shrinkage during the 3 months after RT. Of 24 evaluable cases, ulceration and bleeding were significantly relieved in 13 patients. ${ }^{6}$ Coelho et al retrospectively analyzed 57 LABC individuals who were refractory to primary systemic therapy. RT of $50 \mathrm{~Gy} / 25$ fractions

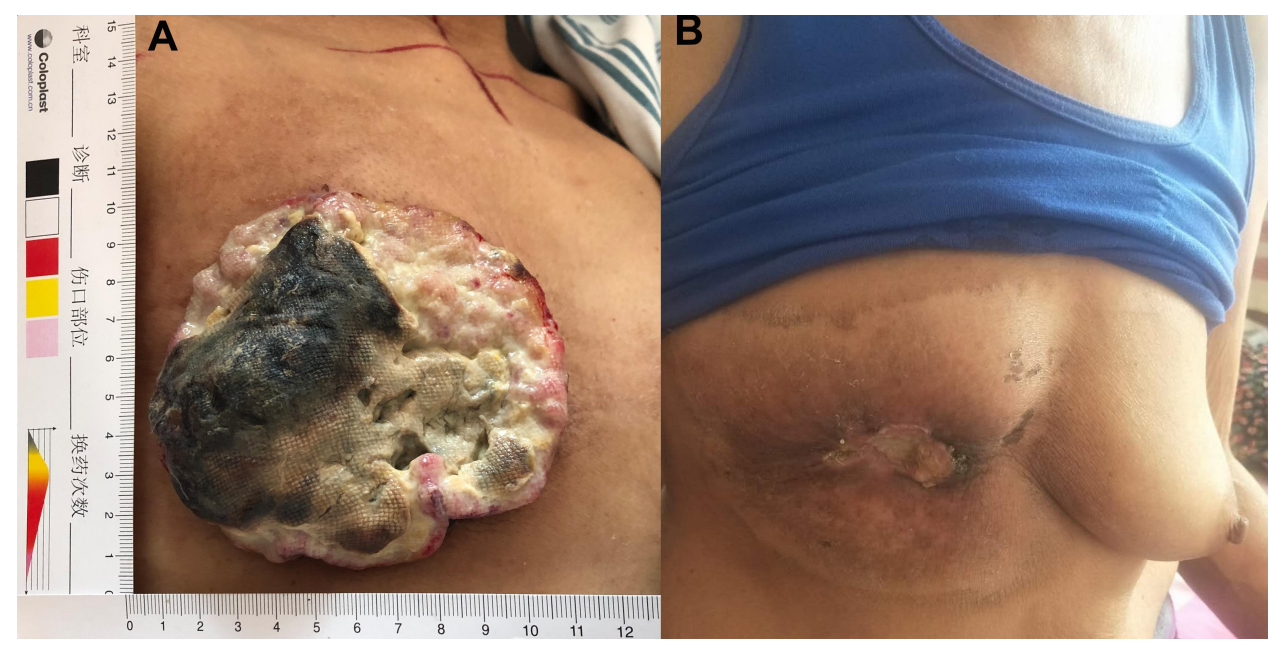

Figure 2 The effect evaluation between pre-RT and 2 months after RT. (A) The fungated, cauliflower-like tumor occupied the entire right breast before RT. (B) The tumor shrank significantly and eventually fell off 2 months after RT. 
downsized the tumors in $75.4 \%$ patients, allowing the performance of mastectomy. Those candidates who were eligible for mastectomy had an obvious overall survival (OS) advantage compared with those who were not eligible (49 vs 18 months). ${ }^{7}$ Kosma et al performed hypofractionated RT, with concurrent 5-fluorouracil (5-FU) treatment, for 17 locally, far-advanced $\mathrm{BC}$ patients and attained an objective response rate (ORR) of $70 \%$. The hypofractionated chemoradiotherapy schema used in this study was as follows: 4-5 Gy per fraction, twice per week; 5 -FU, $300 \mathrm{mg} / \mathrm{m}^{2}$, administered intravenously 1 hour before each RT fraction. ${ }^{8}$ For unresectable cohorts, hypofractionated RT conferred long-term local control, associated with mild side effects and convenience. ${ }^{9}$

Conflicting views have been reported regarding the response of TNBC to RT. Post-mastectomy radiation therapy (PMRT) trials revealed that the absolute decrease in the local relapse rate associated with PMRT in TNBC was $17 \%$, less than the $48 \%$ reported for the luminal B and the $29 \%$ reported for the luminal A subtypes, which was interpreted as an indication of the relative radioresistance of TNBC compared with hormone receptor-positive cohorts. $^{10,11}$ Similarly, among breast-conserving BC candidates, the addition of RT resulted in reduced benefit and local failure reduction among ER-poor individuals. ${ }^{10,12} \mathrm{In}$ contrast, TNBC patients, who are defective in the breast cancer 1 (BRCA1) pathway, were considered to be inherently more radiosensitive. These controversial results can be partly explained by the heterogeneity associated with TNBC, which has diverse subtypes and unclear biological mechanisms. The BRCA1/2 mutation status of our patient was unknown because she refused to undergo BRCA1/2 testing.

In our patient, the tumor was so large and refractory to multiple regimens that RT alone did not achieve an optimal effect. The satisfactory short-term efficacy achieved following RT in our case was the result of two components. First, the hypofractionated scheme of applying 40 Gy/8 fractions achieved better control of tumor. Compared with conventional RT fractions, hypofraction has the advantage of better tumor control. Early-passage cells treated with a hypofractionated irradiation scheme showed less proliferative and invasive abilities. The proportion of tumor stem cells was relatively low, suggesting increased sensitivity to hypofractionated RT. ${ }^{13}$ Second, the administration of apatinib played a synergistic role in combination with RT. Apatinib is a novel, small-molecule tyrosine kinase inhibitor (TKI) that binds to the intracellular segment of VEGFR2, exerting a certain anti-tumor effect in BC. In a retrospective study of 24 multi-drug-resistant, advanced $\mathrm{BC}$ patients, reported by $\mathrm{Lü} \mathrm{er} \mathrm{al,} \mathrm{apatinib}$ monotherapy administered at $500 \mathrm{mg}$ daily achieved an ORR of $41.7 \%$ and a PFS of 4.7 months. ${ }^{14}$ VEGFR2 has been shown to be upregulated greatly In TNBC. ${ }^{15}$ For heavily pretreated, metastatic TNBC individuals, apatinib at $500 \mathrm{mg}$ daily contributed to an ORR of $10.7 \%$ and a median PFS of 3.3 months. ${ }^{3}$ In both studies, apatinib was administered until disease progression or unacceptable toxicity. However, in our patient, the short-term application of apatinib made the local control of tumor and prevention of distant metastasis greatly compromised. Importantly, the inhibition of the VEGF pathway helped to prevent the proliferation of hypoxic cells, which is generally regarded as a critical issue for radioresistance. ${ }^{16,17}$ Additionally, the activation of the VEGF and VEGFR2 pathway resulted in increased vascular cell permeability, followed by increased interstitial fluid pressure, which aggravated hypoxia. Anti-VEGFR2 was revealed to reduce the avidity of endothelial cells after RT, which was closely associated with relapse following RT (Figure 3). ${ }^{16,18,19}$

Certain antiangiogenic agents can transiently normalize the abnormal structures and functionality of the tumor vasculature, increasing the efficiency of oxygen and drug delivery. ${ }^{20,21}$ Therefore, chemotherapy or RT applied during the normalization window will result in increased efficacy. However, determining the correct dose, time, and duration of various antiangiogenic agents is fundamental to inducing a normalization effect. ${ }^{22}$ Apatinib administered at $250 \mathrm{mg}$ daily for 5 days was able to induce vascular normalization in animals. However, this type of vascular normalization effect of apatinib varies from person to person. ${ }^{23}$ In our patient, the application of apatinib (250mg daily for 34 days) before RT might play a role in normalizing tumor blood vessels, which contributed to enhance the RT efficacy to a certain extent.

Unfortunately, our patient died of brain metastasis only 5 months after RT and experienced local failure. She only took apatinib for 4 weeks after RT, without any further treatment. This outcome highlights that a novel and effective systemic treatment strategy is urgently necessary to exert anti-metastatic efficacy and strengthen the role of local treatment. Compared with other subtypes, TNBC is more immunogenic, which indicates that TNBC might obtain additional benefits from immunotherapy. ${ }^{24,25}$ Impassion-130 was the first clinical trial to report the 


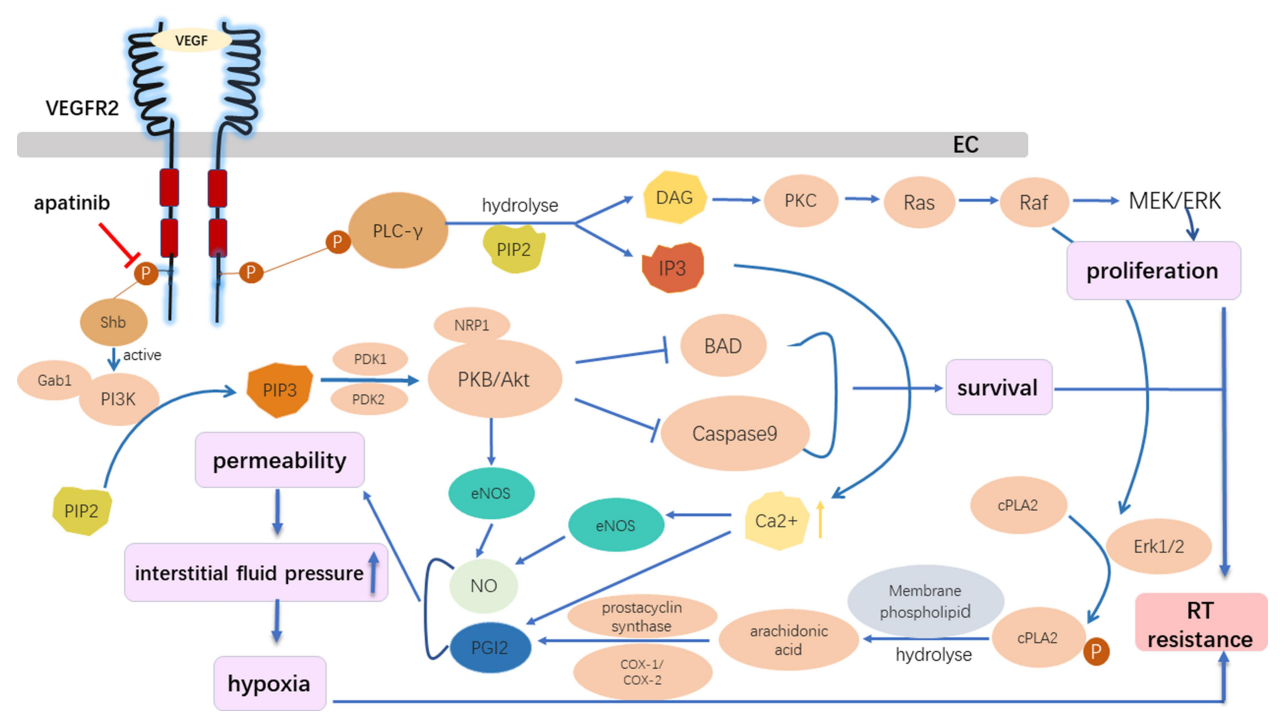

Figure 3 The role of the VEGFR2 inhibitor in the reversal of radioresistance. Apatinib, which acts on the intracellular segment of VEGFR2, inhibited downstream signal activation despite the binding between VEGF with VEGFR2 in vascular endothelial cells, preventing the activation of the following pathways: the Ras/Raf/MEK/ERK pathway, which promotes EC proliferation; and the PI3K/AKT pathway, which inhibits EC apoptosis and promotes EC survival. The activation of VEGF/VEGFR2 could increase the intracellular calcium concentration, activating endothelial nitric oxide synthase, and promoting the production of nitric oxide and prostacyclin. Thus, vascular cell permeability greatly increased, followed by an increase in the interstitial fluid pressure, which is further aggravated by hypoxia. The VEGFR2 inhibitor overcame radiation resistance by inhibiting the proliferation and survival of ECs and reducing hypoxia.

Abbreviations: BAD, Bcl-2 associated death promoter; COX-1/2, cyclooxygenases; DAG, sn-1,2-diacylglycerol; EC, endothelial cell; ERK 1/2, extracellular regulated kinases I and 2; GabI, Grb2-associated binder-I; IP3, inositol (I,4,5)-trisphosphate; eNOS, endothelial nitric oxide synthase; NO, nitric oxide; PDKI/2, phosphoinositide-dependent kinases I and 2; PGI2, prostacyclin; PI3K, phosphoinositide 3-kinase; PIP2, phosphatidylinositol (4,5)-bisphosphate; PIP3, phosphatidylinositol (3,4,5)-trisphosphate; PKB, protein kinase B; PKC, protein kinase C; cPLA2, cytosolic phospholipase A2; PLC- $\gamma$, phospholipase C- $\gamma$; Shb, Src Homology 2 domain-containing adapter protein B.

positive results of immunotherapy for TNBC. Atezolizumab, a programmed death-ligand 1 (PD-L1) inhibitor, has been approved as the first option, combined with Nab-paclitaxel, for the treatment of locally advanced or metastatic PD-L1 ${ }^{+}$TNBC. ${ }^{26,27}$ According to the report by Kim, in a human TNBC xenograft mouse model, the administration of natural killer (NK) cells plus RT dramatically suppressed pulmonary, liver, and lymph node metastases. ${ }^{28}$ Our patient was unable to afford the cost of immunotherapy. According to the study by Jiang et al, transcriptome-based data further classified TNBC into four settings: luminal androgen receptor (LAR); immunomodulatory (IM); basal-like immune-suppressed (BLIS); and mesenchymal-like (MES). ${ }^{29}$ The LAR subtype had a high frequency of mutations associated with cell cycle activation and HER-2, suggesting that a CDK4/6 inhibitor or an anti-HER-2 approach might be important. ${ }^{29}$ Immunotherapy might play a critical role in IM because immunoreactive cells and immune activators are significantly enriched in this subtype. ${ }^{29}$ BLIS demonstrated a high frequency of BRCA1/2 germline mutations and genomic instability. Platinum and poly (ADP-ribose) polymerase (PARP) inhibitors have been shown to be effective for BLIS, with high homologous recombination repair defect scores. ${ }^{29}$ MES is associated with the enrichment of tumor stem cell-related genes and angiogenesis-related pathway activation. Strategies that target tumor stem cells or angiogenesis might work. ${ }^{29}$ For our patient, her refusal to allow a comprehensive analysis of the genomic landscape hampered our ability to understand the nature of her tumor and prescribe individualized and truly effective precision management strategies.

\section{Conclusion}

The treatment of LABC that is refractory to multiple lines of systemic treatment approaches, especially the TNBC subtype, is challenging and requires special attention. Hypofractionated RT plus antiangiogenesis conferred a satisfactory, short-term efficacy with minimal toxicity, as described by our case. This combination is promising due to the synergistic effect and improved RT sensitivity induced by the administration of a VEGF pathway inhibitor, shedding light on this difficult situation. Based on these findings, novel modalities, such as immunotherapy and targeted therapy, that aim to eradicate metastasis and enhance local control are essential for transforming this short-term effect into long-term efficacy. Genomic and 
transcriptomic profiles will deepen our understanding of TNBC and facilitate the tailoring of precision treatments.

\section{Statement of Ethics}

This is a case report and institutional approval was not needed.

\section{Consent for Publication}

Written informed consent was obtained from the patient for publication of this case report and accompanying images.

\section{Acknowledgments}

The present work was supported by the Science and Technology Department of Jilin Province [Grant Nos. 20200201370JC, 20200201506JC].

\section{Disclosure}

The authors report no conflicts of interest in this work.

\section{References}

1. Thakur V, Kutty RV. Recent advances in nanotheranostics for triple negative breast cancer treatment. J Exp Clin Cancer Res. 2019;38 (1):430. doi:10.1186/s13046-019-1443-1

2. Gangi A, Chung A, Mirocha J, Liou DZ, Leong T, Giuliano AE. Breast-conserving therapy for triple-negative breast cancer. JAMA Surg. 2014;149(3):252-258. doi:10.1001/jamasurg.2013.3037

3. Hu X, Zhang J, Xu B, et al. Multicenter Phase II study of apatinib, a novel VEGFR inhibitor in heavily pretreated patients with metastatic triple-negative breast cancer. Int $J$ Cancer. 2014;135 (8):1961-1969. doi:10.1002/ijc.28829

4. Sorlie T, Perou CM, Tibshirani R, et al. Gene expression patterns of breast carcinomas distinguish tumor subclasses with clinical implications. Proc Natl Acad Sci U S A. 2001;98(19):10869-10874. doi:10.1073/pnas.191367098

5. Kassam F, Enright K, Dent R, et al. Survival outcomes for patients with metastatic triple-negative breast cancer: implications for clinical practice and trial design. Clin Breast Cancer. 2009;9(1):29-33. doi:10.3816/CBC.2009.n.005

6. Yee C, Alayed Y, Drost L, et al. Radiotherapy for patients with unresected locally advanced breast cancer. Ann Palliat Med. 2018;7 (4):373-384. doi:10.21037/apm.2018.05.13

7. Coelho RC, Da Silva FML, Do Carmo IML, Bonaccorsi BV, Hahn SM, Faroni LD. Is there a role for salvage radiotherapy in locally advanced breast cancer refractory to neoadjuvant chemotherapy? Breast. 2017;31:192-196. doi:10.1016/j. breast.2016.10.026

8. Kosma L, Koukourakis M, Skarlatos J, et al. Hypofractionated radiotherapy with 5-fluorouracil radiosensitization for locally "far advanced" breast cancer. Am J Clin Oncol. 1997;20(6):562-566. doi:10.1097/00000421-199712000-00005

9. Ghiam AF, Spayne J, Lee J. Current challenges and future perspectives of radiotherapy for locally advanced breast cancer. Curr Opin Support Palliat Care. 2014;8(1):46-52. doi:10.1097/ SPC.000000000000032

10. Moran MS. Radiation therapy in the locoregional treatment of triple-negative breast cancer. Lancet Oncol. 2015;16(3):e113-122. doi:10.1016/S1470-2045(14)71104-0
11. Kyndi M, Sorensen FB, Knudsen H, Overgaard M, Nielsen HM, Overgaard J. Estrogen receptor, progesterone receptor, HER-2, and response to postmastectomy radiotherapy in high-risk breast cancer: the Danish breast cancer cooperative group. J Clin Oncol. 2008;26 (9):1419-1426. doi:10.1200/JCO.2007.14.5565

12. Darby S, McGale P, Correa C, et al. Effect of radiotherapy after breast-conserving surgery on 10-year recurrence and 15-year breast cancer death: meta-analysis of individual patient data for 10,801 women in 17 randomised trials. Lancet. 2011;378(9804):1707-1716.

13. Zhang $\mathrm{H}$, Wan $\mathrm{C}$, Huang J, et al. In vitro radiobiological advantages of hypofractionation compared with conventional fractionation: early-passage NSCLC cells are less aggressive after hypofractionation. Radiat Res. 2018;190(6):584-595. doi:10.1667/ RR14951.1

14. Lu HM, Zhang MW, Niu LM, Zeng HA, Yan M. [Clinical observation of apatinib mesylate for the treatment of multi- drug resistant advanced breast cancer]. Zhonghua Yi Xue Za Zhi. 2018;98 (16):1246- 1249. doi:10.3760/cma.j.issn.0376-2491.2018.16.012. Chinese

15. Gao Z, Shi M, Wang Y, Chen J, Ou Y. Apatinib enhanced anti-tumor activity of cisplatin on triple-negative breast cancer through inhibition of VEGFR-2. Pathol Res Pract. 2019;215(7):152422. doi:10.1016/j.prp.2019.04.014

16. Mortezaee K, Parwaie W, Motevaseli E, et al. Targets for improving tumor response to radiotherapy. Int Immunopharmacol. 2019;76:105847. doi:10.1016/j.intimp.2019.105847

17. Williams KJ, Telfer BA, Shannon AM, Babur M, Stratford IJ, Wedge SR. Inhibition of vascular endothelial growth factor signalling using cediranib (RECENTIN TM; AZD2171) enhances radiation response and causes substantial physiological changes in lung tumour xenografts. $B r \quad J$ Radiol. 2008;81(No special_issue_1):S21-27. doi: $10.1259 / \mathrm{bjr} / 59853976$

18. Ivy SP, Wick JY, Kaufman BM. An overview of small-molecule inhibitors of VEGFR signaling. Nat Rev Clin Oncol. 2009;6 (10):569-579. doi:10.1038/nrclinonc.2009.130

19. Holmes K, Roberts OL, Thomas AM, Cross MJ. Vascular endothelial growth factor receptor-2: structure, function, intracellular signalling and therapeutic inhibition. Cell Signal. 2007;19(10):2003-2012. doi:10.1016/j.cellsig.2007.05.013

20. Jain RK. Normalization of tumor vasculature: an emerging concept in antiangiogenic therapy. Science. 2005;307(5706):58-62. doi:10.1126/ science. 1104819

21. Luo CW, Wu CC, Ch'ang HJ. Radiation sensitization of tumor cells induced by shear stress: the roles of integrins and FAK. Biochim Biophys Acta. 2014;1843(9):2129-2137. doi:10.1016/j. bbamcr.2014.06.007

22. Ma J, Chen CS, Blute T, Waxman DJ. Antiangiogenesis enhances intratumoral drug retention. Cancer Res. 2011;71(7):2675-2685. doi:10.1158/0008-5472.CAN-10-3242

23. Liu M, Li H, Wang X, Jing L, Jiang P, Li Y. Experimental study of the vascular normalization window for tumors treated with apatinib and the efficacy of sequential chemotherapy with apatinib in lung cancer-bearing mice and patients. Cancer Med. 2020;9 (8):2660-2673. doi:10.1002/cam4.2923

24. Akiki M, Haddad FG, Kourie HR, Khaddage A, Smayra VT. PD-L1 an unavoidable biomarker in advanced triple-negative breast cancer. Biomark Med. 2019;13(18):1539-1541. doi:10.2217/bmm-2019-0344

25. Razazan A, Behravan J. Single peptides and combination modalities for triple negative breast cancer. $J$ Cell Physiol. 2020;235 (5):4089-4108. doi:10.1002/jcp. 29300

26. Dolgin E. Atezolizumab Combo Approved for PD-L1-positive TNBC. Cancer Discov. 2019;9(5):OF2. doi:10.1158/2159-8290.CDNB2019-038

27. Schmid P, Adams S, Rugo HS, et al. Atezolizumab and nab-paclitaxel in advanced triple-negative breast cancer. $N$ Engl J Med. 2018;379 (22):2108-2121. doi:10.1056/NEJMoa1809615 
28. Kim KW, Jeong JU, Lee KH, et al. Combined NK cell therapy and radiotherapy exhibit long-term therapeutic and anti-metastatic effects in a human triple negative breast cancer model. Int $J$ Radiat Oncol Biol Phys. 2020;108(1):115-125. doi:10.1016/j.ijrobp.2019.09.041
29. Jiang YZ, Ma D, Suo C, et al. Genomic and transcriptomic landscape of triple-negative breast cancers: subtypes and treatment strategies. Cancer Cell. 2019;35(3):428-440.e5. doi:10.1016/j. ccell.2019.02.001

\section{Publish your work in this journal}

Cancer Management and Research is an international, peer-reviewed open access journal focusing on cancer research and the optimal use of preventative and integrated treatment interventions to achieve improved outcomes, enhanced survival and quality of life for the cancer patient.
The manuscript management system is completely online and includes a very quick and fair peer-review system, which is all easy to use. Visit http://www.dovepress.com/testimonials.php to read real quotes from published authors. 